Revue Sciences/Lettres

Sciences / Lettres

1| 2013

Transferts culturels

\title{
La notion de transfert culturel
}

\section{Michel Espagne}

\section{OpenEdition}

Journals

Édition électronique

URL : http://journals.openedition.org/rsl/219

DOI : $10.4000 /$ rsl. 219

ISSN : 2271-6246

\section{Éditeur}

Éditions Rue d'Ulm

Référence électronique

Michel Espagne, «La notion de transfert culturel », Revue Sciences/Lettres [En ligne], 1 | 2013, mis en ligne le 01 mai 2012, consulté le 10 décembre 2020. URL : http://journals.openedition.org/rsl/219 ; DOI : https://doi.org/10.4000/rsl.219

Ce document a été généré automatiquement le 10 décembre 2020.

(C) Revue Sciences/Lettres 


\title{
La notion de transfert culturel
}

\author{
Michel Espagne
}

1 Tout passage d'un objet culturel d'un contexte dans un autre a pour conséquence une transformation de son sens, une dynamique de resémantisation, qu'on ne peut pleinement reconnaitre qu'en tenant compte des vecteurs historiques du passage. On peut donc dire d'emblée que la recherche sur les transferts culturels concerne la plupart des sciences humaines même si elle s'est développée à partir d'un certain nombre de points d'ancrage précis. Aller au-delà de cette définition minimaliste suppose de fermer un certain nombre de fausses pistes que semble impliquer le vocable lui-même. Transférer, ce n'est pas transporter, mais plutôt métamorphoser, et le terme ne se réduit en aucun cas à la question mal circonscrite et très banale des échanges culturels. C'est moins la circulation des biens culturels que leur réinterprétation qui est en jeu.

2 La notion de transfert culturel s'est développée dans le cadre d'études de l'Allemagne du $\mathrm{XIX}^{\mathrm{e}}$ siècle dans ses liens avec la France ${ }^{1}$. La référence à l'Allemagne tient alors une place structurelle dans le développement des sciences de l'homme. De Victor Cousin, qui établit durablement le cadre de la philosophie française en se réclamant de ses liens avec Hegel ou Schelling, jusqu'à Jacques Offenbach, dont la musique accompagne la fête du Second Empire, l'Allemagne devient un élément constitutif de la vie intellectuelle française. Le positivisme ou le saint-simonisme ne sont pas exempts d'une pénétration qui caractérise les études romanes ou les sciences de l'Antiquitée

3 Pour aborder cette référence, il fallait, d'une part, se rendre compte que la connaissance objective de l'aire culturelle allemande était moins importante que les remaniements auxquels elle pouvait donner lieu et, d'autre part, explorer les vecteurs de la translation. Les transferts culturels se situaient dès lors au point de rencontre d'une recherche de type herméneutique, centrée sur la détermination de sens nouveaux, et d'une enquête historico-sociologique concernant tous les vecteurs de transferts entre les deux pays. On pouvait tout à la fois reconnaître les traces d'une connaissance de Hegel chez les saintsimoniens, observer les reconstructions tout à fait originales auxquelles elles avaient donné lieu et suivre de près la fréquentation des universités prussiennes par ceux qui transmettraient des éléments de savoir. La recherche sur les transferts culturels devait admettre qu'on peut s'approprier un objet culturel et s'émanciper du modèle qu'il 
constitue, c'est-à-dire qu'une transposition, aussi éloignée soit-elle, a autant de légitimité que l'original. En conséquence, dans les sciences humaines et sociales, la comparaison comme principe additionnel d'ouverture à des espaces différents perdait de son intérêt et devait être relayée par l'observation des formes de métissage et d'hybridité 3 . Penser en termes de transferts culturels conduisait ainsi à relativiser la pertinence de la comparaison. Celle-ci tend en effet à opposer des entités pour comptabiliser leurs ressemblances et leurs dissemblances, mais elle ne tient guère compte de l'observateur qui compare, oppose pour rassembler, projette son propre système de catégories, crée les oppositions qu'il réduit et appartient lui-même en général à l'un des deux termes de la comparaison. Fondé notamment sur la grammaire comparée des langues indoeuropéennes, le comparatisme en épouse les limites. Il semble tout particulièrement délicat d'aborder par le biais d'une histoire comparée les territoires extra-européens et, de façon générale, de mettre en relation des territoires, des cultures ou des littératures entre lesquels une différence qualitative radicale est implicitement présupposée.

De même, la catégorie de l'influence, dont l'étymologie suffit à montrer la dimension magique, devait être remplacée par une approche critique des contacts historiquement constatables et des adaptations ou réinterprétations auxquelles ces contacts avaient donné lieu. Il convenait également de faire l'économie des concepts d'authenticité dans la transmission ou de supériorité de l'original sur la copie. On sait qu'un disciple secondaire de Schelling, Krause, a donné lieu dans le monde hispanique à une école de pensée, le " krausismo ", qui relègue dans l'ombre le reste de la philosophie allemande et repose sur une connaissance au fond assez sommaire des textes. Il n'en reste pas moins que cette forme de pensée libérale, marquant accessoirement un glissement disciplinaire de la métaphysique schellingienne à une pensée politique, est tout aussi légitime que l'impulsion qui lui a donné naissance. On ne mesure pas le krausisme à son degré de fidélité à Schelling, pas plus qu'on ne juge la traduction de Sophocle par Hölderlin au degré d'exactitude avec lequel les segments textuels ont été transposés. À la limite, la connaissance d'une tradition précédant son importation et sa reconstruction peut être extrêmement succincte.

5 Tous les groupes sociaux susceptibles de passer d'un espace national ou linguistique ethnique ou religieux à l'autre peuvent être vecteurs de transferts culturels. Les commerçants transportant des marchandises ont toujours véhiculé également des représentations ou des savoirs. Les traducteurs, les enseignants spécialistes d'une aire culturelle étrangère, les émigrés politiques, économiques ou religieux, les artistes répondant à des commandes, les mercenaires, constituent autant de vecteurs de transferts, et il convient de tenir compte de leurs différentes médiations. Toutefois, on peut fort bien se représenter aussi des transferts reposant sur la circulation d'objets comme des livres ou des œuvres d'art. L'histoire des bibliothèques, de la constitution des fonds étrangers, de la diffusion des produits éditoriaux et de la traduction, comme l'histoire des collections et du marché transnational de l'art, font évidemment partie de la recherche sur les transferts culturels. Et lorsqu'on passe des médiations humaines aux médiations associées à des livres ou à des archives, la question des transferts culturels rencontre celle de la mémoire. En effet, les bibliothèques ou les archives, dont les modes d'organisation méritent souvent qu'on en établisse l'histoire, tendent à conforter des identités. Elles sont généralement organisées suivant un principe de pertinence qui correspond aux représentations de l'identité d'un groupe, la plupart du temps national. Devenir attentif aux transferts culturels implique de réviser, au moins de façon virtuelle, 
les structures de la mémoire collective des bibliothèques et des archives en recherchant des éléments importés, souvent marginalisés. Au demeurant, la mobilisation d'un élément de mémoire étrangère n'a rien de fortuit. Lorsqu'on va chercher dans les strates de la mémoire un élément étranger au contexte d'accueil, c'est en général pour répondre à une constellation de ce même contexte d'accueil. Il convient de distinguer entre mémoire accumulée, encore inutilisée, et mémoire effective.

Un transfert culturel n'a jamais lieu seulement entre deux langues, deux pays ou deux aires culturelles : il y a quasiment toujours des tiers impliqués. On doit donc plutôt se représenter les transferts culturels comme des interactions complexes entre plusieurs pôles, plusieurs aires linguistiques. On fait du passage en Allemagne des Lumières françaises un phénomène bien étroit si on néglige ses racines anglaises et ses prolongements russes. Et aborder la culture de la Russie à l'époque de Catherine II, c'est comprendre l'interaction entre l'Empire russe, la culture allemande de l'impératrice, son intérêt pour la France et pour une Italie souvent revue à travers le prisme de la littérature française. Toutefois, s'il est facile de reconnaître des lieux où se rencontrent de nombreux espaces culturels, des lieux qu'on pourrait considérer, en utilisant un néologisme, comme des "portails de globalisation », la description ne peut s'opérer que sur des rencontres d'un nombre réduit de termes. La représentation de croisements généralisés reste inopérante.

7 Même lorsqu'on aborde un transfert entre deux espaces culturels, on ne peut en aucune manière les considérer chacun comme homogènes et originels : chacun est lui-même le résultat de déplacements antérieurs; chacun a une histoire faite d'hybridations successives. Il convient de garder cela à l'esprit quand on s'efforce de décrire par exemple un transfert culturel franco-allemand. Ni l'Allemagne ni la France ne sont des essences. Toutefois, aussi discutables que soient ces entités, la nécessité d'une description oblige à supposer pour un moment fugitif l'existence d'un système qu'on baptisera Allemagne ou France, hellénisme ou latinité. Mais on s'attachera immédiatement à montrer que ces entités sont élaborées à partir d'importations. La France c'est l'Allemagne, comme la latinité est grecque ou la scolastique médiévale est arabe, le bouddhisme chinois est indien, etc. Les aires culturelles, dont la recherche sur les transferts culturels révèle les imbrications, sont donc des configurations provisoires, mais nécessaires à la compréhension des phénomènes de circulation culturelle.

De façon générale, les sciences humaines correspondent à des récits nationaux, limités à des espaces linguistiques particuliers. Elles fabriquent des identités à partir d'importations et des reformulations qui les accompagnent. La révision systématique de ces constructions identitaires offre aux recherches sur les transferts culturels un vaste terrain d'investigations, dont l'horizon serait une histoire transnationale des sciences humaines.

9 Même si la philosophie, à l'instar des mathématiques, revendique une universalité de principe, une indépendance des concepts vis-à-vis des langues qui les véhiculent, il apparait qu'elle s'est développée durant le long $\mathrm{XIX}^{\mathrm{e}}$ siècle avec une référence allemande, l'idéologie laïque de la $\mathrm{III}^{\mathrm{e}}$ République se réclamant d'un kantisme renouvelé. Naturellement, ni le Schelling de Ravaisson ni le Hegel de Kojève ne correspondent aux originaux dont leurs interprètes se réclament. Il est du plus grand intérêt de déterminer ce que leur interprétation ajoute, mais il faut surtout observer qu'une tradition intellectuelle prétendant constituer une morale civique nationale se construit à partir de références importées. La phénoménologie serait difficilement pensable sans une 
importation de Husserl précédant d'autres formes de circulation de ce qui s'est élaboré sur le fondement de cette référence. En cela, la philosophie française n'est d'ailleurs pas unique, et Martial Guéroult s'était déjà attaché à mettre en lumière tout ce que Fichte devait à des discours de la Révolution française, à des prises de positions politiques exprimées en France à son époque. Sans doute la tendance d'une frange significative de la philosophie française contemporaine à exprimer en anglais une " philosophie de l'esprit » résultant dans une large mesure de la traduction anglaise de la philosophie autrichienne correspond-elle au même schéma de construction d'une identité se voulant universelle à partir d'un système de références à une culture importée - dont les concepts centraux ont d'ailleurs des ancrages linguistiques largement ignorés.

10 En histoire de l'art, un modèle de transfert est fourni tant par l'application à l'Italie de l'herméneutique allemande que par la recherche d'éléments d'une strate chronologiquement antérieure dans une strate ultérieure (transferts diachroniques, par exemple, le Moyen Âge dans la Renaissance). Lorsque Heinrich Wölfflin applique à l'Italie ses concepts fondamentaux de l'histoire de l'art, il ne fait rien d'autre que transposer des catégories de la psychologie allemande à un autre espace ${ }^{4}$. Carl Justi ne procède pas très différemment lorsqu'il applique à l'art de Velázquez, ou plutôt au phénomène de Velázquez dans son temps, des catégories apparentées à l'herméneutique de Wilhelm Dilthey ${ }^{5}$. Quand Anton Springer observe dans l'art médiéval allemand des traces de l'Antiquité réinterprétées selon les besoins d'un contexte nouveau, il ne fait rien d'autre qu'analyser un transfert culturel, et ses observations serviront de modèle à Aby Warburg, qui le lit durant son séjour à Florence ${ }^{6}$.

11 L'anthropologie en tant que science est particulièrement attentive aux relations entre les cultures, à leurs contacts et aux formes d'interpénétration qui leur impriment une dynamique. Il est tout à fait notable que l'un des fondateurs de l'anthropologie américaine, Franz Boas, était un Allemand de Minden émigré aux États-Unis, où il appliqua à l'étude des Indiens de la côte ouest, les Kwakiutl notamment, des modes d'approche démarqués des collections de chants populaires des frères Grimm. Il s'agissait de rassembler des récits en langue indienne, de les transcrire pour la première fois avant de procéder à leur analyse et de reconnaître ce qu'ils dévoilaient des sociétés indiennes. Et lorsqu'il étudiait les contaminations entre ethnies, Boas cherchait à observer des contacts historiquement reconnaissables pour justifier les récurrences systémiques, les homologies structurelles. Il ne cherchait pas à comparer, mais à observer la genèse d'imbrications, à suivre des contaminations. Non seulement le parcours biographique et intellectuel de Boas est un cas notable de transfert culturel, mais la méthode qu'il développe est tout particulièrement adaptée à ce phénomène. Et non seulement Boas incarne de façon suffisamment éclatante l'idée de transfert culturel en anthropologie pour qu'une analyse attentive de son œuvre sous cet angle s'impose, mais nombre de ses proches disciples sont des émigrants venus de l'Europe germanique et participant à un vaste déplacement vers les États-Unis de connaissances acquises ailleurs. De façon générale, l'idée, véhiculée par Boas, selon laquelle la langue est le principal marqueur d'une culture est un héritage de Humboldt, dont on peut suivre les traces à travers l'Europe et notamment en Russie où, par la médiation de Heymann Steinthal et de certains élèves slaves, elle participe au socle sur lequel repose le formalisme linguistique et anthropologique. Lorsque Lévi-Strauss, qui s'y rattache par Jakobson notamment, vient en Amérique et rencontre Boas, deux formes de transferts fondamentaux en anthropologie convergent. 

dans les librairies, sont toujours considérées comme un élément extérieur, légèrement périphérique); sur des figures de l'articulation entre les traditions littéraires, sur les panthéons étrangers que chaque culture se construit (après Eugène-Melchior de Vogüé, Tolstoï est devenu, sinon un écrivain français, du moins un écrivain central dans le panthéon français des littératures étrangères), sur les écrivains utilisant une langue qui n'est pas la leur (du Turc de langue allemande Feridun Zaimoglou au Russe de langue française Andrei Makine en passant par le Somalien de langue anglaise Nuruddin Farah). Dans cette perspective, l'histoire littéraire peut être revue du point de vue de continuités alternatives ne tenant pas seulement compte d'éléments nationaux. Stefan George étant profondément inspiré par Mallarmé, qu'il traduit, ou Hölderlin par Rousseau, on ne devrait pas tenter de reconstruire des filiations dans l'histoire du lyrisme en Allemagne qui ne tiendraient pas compte de ces apports extérieurs. Mais André Chénier et Schiller seraient pour les mêmes raisons des moments de la littérature russe, et l'ensemble des histoires littéraires devrait dès lors être réécrit. est déterminée par les sciences des aires culturelles proches, par exemple la romanistique allemande ou la slavistique française. Ces sciences résultent d'un compromis entre l'espace littéraire étudié et l'horizon propre de celui qui l'observe. En outre, elles peuvent faire l'objet d'importations. Si on considère par exemple que la romanistique allemande telle que l'incarnait Friedrich Christian Diez est, par son approche même d'une globalité des cultures romanes, marquée par une conception des objets de la philologie clairement empruntée à une tradition allemande, son importation en France par Gaston Paris, qui l'applique de façon privilégiée au Moyen Âge français, correspond à une réinterprétation de son objet même, la « romania ».

14 L'examen des histoires littéraires sous l'angle des transferts culturels dont elles constituent le témoignage est d'autant plus significatif que, dans tous les pays européens, elles font partie intégrante de la construction des nations. Depuis la Chanson de Roland jusqu'au Dit du prince Igor, il n'est pas de nation européenne qui existe sans ce texte fondateur - qu'on trouve dans des espaces lointains (par exemple l'Histoire de Kieu pour la littérature vietnamienne, Le Chevalier à la peau de panthère pour la Géorgie et même dans l'Antiquité avec l'épopée de Gilgamesh). Or il est bien évident que ces constructions sont fréquemment liées à des importations étrangères. Le Kalevipoeg de Kreutzvald, épopée nationale estonienne, est le résultat du travail de philologues formés à une tradition allemande, post-herdérienne, qu'on sent également à l'œuvre dans la grande épopée finnoise que fut le Kalevala de Lönnrot. Il y a des restes de poésie ossianique dans l'interprétation que livre Friedrich August Wolf de l'épopée homérique, dont il fait l'œuvre de tout un peuple. À partir d'Ossian et de Herder, le modèle de l'œuvre fondant la nation s'est imposé à travers toute l'Europe. La révision de l'histoire littéraire sous l'angle des transferts culturels permet de mettre en évidence la circulation des modèles censés fonder des littératures nationales.

Certaines sciences humaines transcendent à vrai dire d'emblée les cadres nationaux. C'est par exemple le cas de l'orientalisme, tel qu'il a pu se développer depuis le début du XIX ${ }^{e}$ siècle: une revue comme le Journal asiatique peut alors apparaître comme un organe franco-allemand d'exploration des littératures et cultures du Proche-Orient, en particulier l'arabe, le turc et le persan. Figure centrale de cette nouvelle discipline, Silvestre de Sacy a été aussi en matière d'études orientales une sorte de "praeceptor 
Germaniae ", puisque la plupart des titulaires de chaires d'études orientales de la première moitié du XIX ${ }^{e}$ siècle ont été ses élèves. Tout se passe comme si la France et l'Allemagne, auxquelles on pourrait facilement ajouter l'Angleterre, l'Italie et la Russie, abandonnant l'ambition de construire des sciences séparées de l'Orient, avaient cherché à en avoir une perception commune, reposant elle-même sur un compromis entre, d'une part, leur objet dans toute sa complexité et sa diversité et, d'autre part, leur propre cadre conceptuel.

La recherche sur les transferts fait partie des historiographies culturelles transnationales. Mais elle n'est pas pour autant réduite à l'analyse des imbrications entre espaces nationaux de l'Europe moderne. Elle peut fort bien trouver d'autres terrains d'application. L'appropriation par la cour de l'empereur de Chine des mathématiques européennes telles qu'elles ont été transmises par les missionnaires jésuites pour devenir en Extrême-Orient un signe de pouvoir correspond pleinement à un phénomène de transfert culturel, tout comme le rôle joué par la Prusse dans la réforme de la société japonaise à l'ère du Meiji. Dans l'Antiquité, plus encore qu'à l'époque moderne, il est habituel de considérer l'Empire romain ou le monde grec comme des entités homogènes. Or l'archéologie a depuis longtemps observé des phénomènes de métissage; elle est à la recherche de passages difficiles à cerner entre l'Égypte et la Grèce, entre les mathématiques mésopotamiennes et les mathématiques grecques. Un changement de perspective pourrait amener à considérer l'Antiquité classique comme un cadre de réappropriations en chaîne d'éléments propres aux cultures entourant la Méditerranée. Sur les côtes de la Turquie actuelle, les cités grecques étaient aussi peuplées de Cariens, de Lyciens ou de Lydiens acculturés, et les traces archéologiques de villes hittites d'Anatolie montrent la présence de colonies étrangères mésopotamiennes. Les imbrications ne sont pas au demeurant à sens unique. La rencontre des cultures de l'Antiquité classique avec les peuples situés à leur périphérie a aussi permis l'émergence d'entités nouvelles, de la Bactriane ou de la Sogdiane gréco-irano-bouddhiques ${ }^{7}$ à la culture gallo-romaine.

Que l'histoire archaïque des sociétés sur lesquelles se fonde l'auto-perception de l'Europe moderne soit faite de transferts, cela a déjà légitimé des remises en cause de la domination européenne. L'ouvrage de Cheikh Anta Diop sur l'Égypte noire (Nations nègres et culture, 1954), conforté par les hypothèses de Black Athena de Martin Bernal, visait essentiellement à désigner, au début de la décolonisation, un héritage africain archaïque au cœur de la civilisation européenne. La question des transferts culturels peut donc inclure une partie des approches postcoloniales. Mais elle ne saurait s'y réduire. Quand un médiéviste met en évidence le fait que la mystique allemande incarnée par maître Eckhart emprunta sa théorie de l'intellect à Averroès ${ }^{8}$, et que le détour de la philosophie grecque par la pensée islamique est devenu un objet classique des études grecques ${ }^{9}$, il n'est pas question de post-colonialisme, mais bien de circulation de systèmes conceptuels qui, en fonction du contexte d'accueil, modifient leur signification ${ }^{10}$.

18 L'historiographie des transferts culturels relativise tout particulièrement la notion de centre. Il est clair que l'histoire, dès qu'elle dépasse les limites de la nation ou de l'aire culturelle dont elle est l'émanation pour intégrer des cercles concentriques plus larges, considère que les références propres à son aire culturelle d'appartenance sont centrales. Lorsque des histoires universelles commencent à être écrites à l'Université de Göttingen, dans le dernier tiers du XVIII ${ }^{e}$ siècle ${ }^{11}$, et que l'Arabie, l'Inde ou la Chine sont intégrées dans ce balayage global de l'histoire humaine, on considère que l'Europe est au centre, et qu'en entrant dans l'histoire, les cultures périphériques s'intègrent dans un ensemble 
dont le centre est clairement déterminé : européen. On ne peut s'empêcher de penser que la "global history» est elle-même structurée à partir d'un centre anglo-saxon. D'autres centres ont pu exister dans le cours de l'histoire. On pense à l'Empire du milieu et à l'exigence des empereurs chinois en matière de cartographie: que la chine soit représentée au milieu des cartes du monde connu. On pense aux habitudes de la Turquie kémaliste de dessiner des cartes du monde mettant au centre l'Anatolie, elle-même au milieu d'un monde turc équidistant d'un côté de l'Algérie et de l'autre côté de l'Asie centrale $^{12}$. La remise en cause des centres est un élément fondamental de la recherche sur les transferts culturels.

19 La relativité radicale du centre de perspective aboutit à faire coïncider le global et le particulier, chaque particularité devant être créditée de son accès propre au global. Il est des lieux où cette coïncidence est facilitée: des centres urbains, des universités, des bibliothèques, qu'on peut considérer comme des " portails sur la globalité ». Étudier ces lieux (dont on se gardera de donner une liste limitative) constitue évidemment une tâche importante dans la recherche sur les transferts. On peut penser à un lieu comme la bibliothèque de Göttingen, principale bibliothèque des pays germaniques et premier centre à collectionner dès le milieu du XVIII ${ }^{\mathrm{e}}$ siècle toute la littérature scientifique de langue française, anglaise, italienne, russe ou allemande, afin d'en nourrir un enseignement qui se voulait une science universelle de l'homme. De façon assez différente on peut songer à des villes où se rencontrent un grand nombre de groupes ethniques, comme Vilnius/Wilna/Wilno, ville juive, allemande, polonaise, lituanienne, karaite et russe à la fois, lieu de dissémination de la culture juive et lieu d'émergence d'une littérature nationale polonaise puis lituanienne. Ces "portails sur la globalité » associent les transferts culturels à la catégorie du lieu.

Un transfert culturel est parfois une traduction. Il suffit de mettre en regard une édition d'un roman dans une langue quelconque et sa traduction dans une autre langue, d'observer le discours d'accompagnement des quatrièmes de couverture, les illustrations, les formats, l'effet de contexte des séries et même la typographie, pour voir qu'une traduction n'est en aucun cas un équivalent. Elle l'est encore moins lorsqu'elle ne se déclare pas telle mais s'inspire simplement d'un original à la manière dont certains auteurs latins ont pu s'inspirer d'originaux grecs. Lucrèce n'est certainement pas un équivalent de Démocrite. La traduction met en évidence le fait que les concepts sont enracinés dans des contextes sémantiques et que le déplacement de contexte sémantique lié à la traduction représente une nouvelle construction de sens. Mais la traduction est aussi un objet de recherches ressortissant à la sociologie historique ou à l'histoire du livre, lesquelles s'appuient évidemment sur les recherches relatives aux transferts culturels. L'étude prosopographique des traducteurs conduit à s'interroger sur les modes d'acquisition des langues, sur les critères à partir desquels s'opère le choix des livres à transposer. Il importe d'analyser la stratégie des maisons d'édition, leur mode de fonctionnement, l'écho rencontré par les ouvrages traduits. De la traduction des Septante aux premières traductions de Kant au $\mathrm{XIX}^{\mathrm{e}}$ siècle, qui croisaient à grand peine une approche du texte allemand, l'hermétique version latine de Friedrich Gottlieb Born et la version italienne de Vincenzo Mantovani, l'analyse non linguistique du phénomène des traductions est un des axes de la recherche sur les transferts culturels.

21 Cette recherche offre un accès à la compréhension, sinon de l'histoire globale, du moins de configurations transnationales larges, en articulant la description du particulier et de l'universel. La globalité doit en effet être observée à partir de cas bien précis, voire de 
singularités. L'acribie du philologue ou du généticien observant la succession de deux strates textuelles, de deux variantes, accompagne et corrige la réflexion sur la circumnavigation des notions. Aucun planisphère ne peut faire l'économie du dessin des fleuves et des côtes. C'est de la pluralité des langues que peuvent se déduire les déplacements sémantiques des traductions. Davantage une théorie en progrès qu'un essai de doctrine, la recherche sur les transferts culturels pourrait aboutir à un nouveau point de vue sur les sciences humaines et sociales.

\section{NOTES}

1. Voir Michel Espagne et Michael Werner (textes réunis et présentés par), Transferts. Les relations interculturelles dans l'espace franco-allemand (XVIII ${ }^{e}$-XIX ${ }^{e}$ siècles), Paris, Éditions Recherche sur les Civilisations, 1988 ; Michel Espagne, Les Transferts culturels franco-allemands, Paris, PUF, 1999. Voir aussi : http://geschichte-transnational.clio-online.net/transnat.asp

2. C'est parmi des saint-simoniens qu'on trouve les premiers élèves français de Hegel. La référence aux sciences allemandes de l'Antiquité dans la France du XIx ${ }^{\mathrm{e}}$ siècle se reconnaît par exemple à la monumentale entreprise de traduction de la Symbolique de Creuzer par Joseph Daniel Guigniaut (1825-1851).

3. Par métissage il convient d'entendre la resémantisation liée à la rencontre de deux entités culturelles qui elles-mêmes résultent de rencontres et de resémantisations antérieures. Voir Jean-Loup Amselle, Branchements. Anthropologie de l'universalité des cultures, Paris, Flammarion, 2001.

4. Heinrich Wölfflin, Kunstgeschichtliche Grundbegriffe, Munich, Bruckmann, 1915.

5. Carl Justi, Diego Velazquez und sein Jahrhundert, Bonn, Cohen, 1903.

6. Anton Springer, Bilder aus der neueren Kunstgeschichte, Bonn, A. Marcus, 1867.

7. Certaines langues mortes d'Asie centrale (sogdien, tokharien) ne sont quasiment attestées que par des corpus de traductions à partir d'autres langues.

8. Kurt Flasch, D’Averroès à Maître Eckhart. Les sources arabes de la "mystique » allemande, Paris, Vrin, 2008.

9. Philippe Büttgen, Alain de Libera, Marwan Rashed, Irène Rosier-Catach (éd.), Les Grecs, les Arabes et nous. Enquête sur l'islamophobie savante, Paris, Fayard, 2009.

10. Les clefs platoniciennes utilisées par Garcilaso de la Vega pour décrire sa culture originelle aboutissent à une double projection : celle de catégories platoniciennes sur l'histoire du peuple inca, celle d'exemples incas pour conforter un modèle platonicien. Voir Carmen Bernand, Un Inca platonicien. Garcilaso de la Vega, Paris, Fayard, 2005.

11. Luigi Marino, Praeceptores Germaniae: Göttingen 1770-1820, Göttingen, Vandenhoeck \& Ruprecht, 1995 ; Hans-Erich Bödeker, Philippe Büttgen et Michel Espagne (éd.), Göttingen vers 1800. L'Europe des sciences de l'homme, Paris, Le Cerf, 2010.

12. Étienne Copeaux, Une vision turque du monde à travers les cartes de 1931 à nos jours, Paris, CNRS Éditions, 2000. 


\section{RÉSUMÉS}

Tout passage d'un objet culturel d'un contexte dans un autre a pour conséquence une transformation de son sens, une dynamique de resémantisation, qu'on ne peut pleinement reconnaître qu'en tenant compte des vecteurs historiques du passage. On peut donc dire d'emblée que la recherche sur les transferts culturels concerne la plupart des sciences humaines même si elle s'est développée à partir d'un certain nombre de points d'ancrage précis. L'étude des transferts culturels conduit à relativiser l'importance de la comparaison et, par dessus tout, la notion de centre.

Any cultural object passing from one context into another results in a transformation of its meaning and the dynamic of re-semantisation it undergoes can only be fully recognised by taking into account the historical vectors of that transfer. We can therefore say that research dealing with cultural transfers concerns most of the human sciences even though its development was first rooted in specific domains. The study of cultural transfers forces us to downplay the notions of comparison, and, above all, of centre.

\section{INDEX}

Mots-clés : histoire des sciences humaines, transfert culturel, historiographies transnationales Keywords : history of human sciences, cultural transfer

\section{AUTEUR}

\section{MICHEL ESPAGNE}

Directeur de recherche CNRS, Directeur de l'UMR 8547 Pays germaniques : transferts culturels/ archives Husserl. Responsable de l'équipe "Transferts culturels" Parmi les publications : Les Transferts culturels franco-allemands, Paris, PUF, 1999. L'Histoire de l'art comme transfert culturel, Paris, Belin, 2009. Avec Michael Werner (textes réunis et présentés par), Transferts. Les relations interculturelles dans l'espace franco-allemand (XVIII ${ }^{e}$-XIX ${ }^{e}$ siècles), Paris, Éditions Recherche sur les Civilisations, 1988. Avec Hans-Erich Bödeker et Philippe Büttgen (éd.), Göttingen vers 1800. L'Europe des sciences de l'homme, Paris, Le Cerf, 2010. 